\title{
Reactor rate modulation oscillation analysis with two detectors in Double Chooz
}

\section{DOUBLE}

\section{The Double Chooz collaboration}

T. Abrahão, ${ }^{e, d}$ H. Almazan, ${ }^{o}$ J.C. dos Anjos, ${ }^{e}$ S. Appel, ${ }^{v}$ J.C. Barriere, ${ }^{k}$ I. Bekman, ${ }^{a}$ T.J.C. Bezerra, ${ }^{r}, 1$ L. Bezrukov, ${ }^{j}$ E. Blucher, ${ }^{g}$ T. Brugière, ${ }^{q}$ C. Buck, ${ }^{o}$ J. Busenitz, ${ }^{b}$ A. Cabrera, ${ }^{d, a a, 2}$ M. Cerrada, ${ }^{h}$ E. Chauveau, ${ }^{f}$ P. Chimenti, ${ }^{e, 3}$ O. Corpace, ${ }^{k}$ J.V. Dawson, ${ }^{d}$ Z. Djurcic, ${ }^{c}$ A. Etenko, ${ }^{n}$ H. Furuta, ${ }^{s}$ I. Gil-Botella, ${ }^{h}$ A. Givaudan, ${ }^{d}$ H. Gomez, ${ }^{d, k}$ L.F.G. Gonzalez, ${ }^{y}$ M.C. Goodman, ${ }^{c}$ T. Hara, ${ }^{m}$ J. Haser, ${ }^{o}$ D. Hellwig, ${ }^{a}$ A. Hourlier, ${ }^{d, 4}$ M. Ishitsuka, ${ }^{t, 5}$ J. Jochum, ${ }^{w}$ C. Jollet, ${ }^{f}$ K. Kale, ${ }^{f, q}$ M. Kaneda, ${ }^{t}$ M. Karakac, ${ }^{d}$ T. Kawasaki, ${ }^{l}$ E. Kemp,${ }^{y}$ H. de Kerret, ${ }^{d, 6}$ D. Kryn, ${ }^{d}$ M. Kuze, ${ }^{t}$ T. Lachenmaier, ${ }^{w}$ C.E. Lane, ${ }^{i}$ T. Lasserre,${ }^{k, d}$ C. Lastoria, ${ }^{h}$ D. Lhuillier, ${ }^{k}$ H.P. Lima Jr., ${ }^{e}$ M. Lindner, ${ }^{o}$ J.M. López-Castaño, ${ }^{h, 13}$ J.M. LoSecco, ${ }^{p}$ B. Lubsandorzhiev, ${ }^{j}$ J. Maeda, ${ }^{u, m}$ C. Mariani, ${ }^{z}$ J. Maricic, ${ }^{i, 7}$ J. Martino, ${ }^{r}$ T. Matsubara, ${ }^{u, 8}$ G. Mention, ${ }^{k}$ A. Meregaglia, ${ }^{f}$ T. Miletic, ${ }^{i, 9}$ R. Milincic, ${ }^{i, 7}$ A. Minotti, ${ }^{k, 10}$ D. Navas-Nicolás, ${ }^{d, h, 2}$ P. Novella, ${ }^{h, 11}$ L. Oberauer, ${ }^{v}$ M. Obolensky, ${ }^{d}$ A. Onillon, ${ }^{k}$ A. Oralbaev, ${ }^{n}$ C. Palomares, ${ }^{h}$ I.M. Pepe, ${ }^{e}$ G. Pronost, ${ }^{r}, 12$ J. Reichenbacher, ${ }^{b, 13}$ B. Reinhold, ${ }^{o, 7}$ S. Schönert, ${ }^{v}$ S. Schoppmann, ${ }^{o}$ L. Scola, ${ }^{k}$ R. Sharankova, ${ }^{t}$ V. Sibille, ${ }^{k, 4}$ V. Sinev, ${ }^{j}$ M. Skorokhvatov, ${ }^{n}$ P. Soldin, ${ }^{a}$ A. Stahl, ${ }^{a}$ I. Stancu, ${ }^{b}$ L.F.F. Stokes, ${ }^{w}$ F. Suekane,${ }^{s, d}$ S. Sukhotin, ${ }^{n}$ T. Sumiyoshi, ${ }^{u}$ Y. Sun, ${ }^{b, 7}$ C. Veyssiere, ${ }^{k}$ B. Viaud, ${ }^{r}$ M. Vivier, ${ }^{k}$ S. Wagner, ${ }^{d, e}$ C. Wiebusch, ${ }^{a}$ G. Yang ${ }^{c, 14}$ and F. Yermia ${ }^{r}$

\footnotetext{
${ }^{1}$ Now at Department of Physics and Astronomy, University of Sussex, Falmer, Brighton, United Kingdom.

${ }^{2}$ Now at IJC Laboratory, CNRS/IN2P3, Université Paris-Saclay, Orsay, France.

${ }^{3}$ Now at Universidade Estadual de Londrina, 86057-970 Londrina, Brazil.

${ }^{4}$ Now at Massachusetts Institute of Technology, Cambridge, Massachusetts 02139, U.S.A.

${ }^{5}$ Now at Tokyo University of Science, Noda, Chiba, Japan.

${ }^{6}$ Deceased.

${ }^{7}$ Now at Physics \& Astronomy Department, University of Hawaii at Manoa, Honolulu, Hawaii, U.S.A.

${ }^{8}$ Now at High Energy Accelerator Research Organization (KEK), Tsukuba, Ibaraki, Japan.

${ }^{9}$ Now at Physics Department, Arcadia University, 450 S. Easton Road, Glenside, PA 19038, U.S.A.

${ }^{10}$ Now at LAPP, CNRS/IN2P3, 74940 Annecy-le-Vieux, France.

${ }^{11}$ Now at Instituto de Física Corpuscular, IFIC (CSIC/UV), 46980 Paterna, Spain.

${ }^{12}$ Now at Kamioka Observatory, ICRR, University of Tokyo, Kamioka, Gifu 506-1205, Japan.

${ }^{13}$ Now at South Dakota School of Mines \& Technology, 501 E. Saint Joseph St. Rapid City, SD 57701, U.S.A.

${ }^{14}$ Now at State University of New York at Stony Brook, Stony Brook, NY, 11755, U.S.A.
} 
${ }^{a}$ III. Physikalisches Institut, RWTH Aachen University, 52056 Aachen, Germany

${ }^{b}$ Department of Physics and Astronomy, University of Alabama,

Tuscaloosa, Alabama 35487, U.S.A.

${ }^{c}$ Argonne National Laboratory, Argonne, Illinois 60439, U.S.A.

${ }^{d}$ APC, Université de Paris, CNRS, Astroparticule et Cosmologie, F-75006, Paris

${ }^{e}$ Centro Brasileiro de Pesquisas Físicas, Rio de Janeiro, RJ, 22290-180, Brazil

${ }^{f}$ Université de Bordeaux, CNRS/IN2P3, CENBG, F-33175 Gradignan, France

${ }^{g}$ The Enrico Fermi Institute, The University of Chicago, Chicago, Illinois 60637, U.S.A.

${ }^{h}$ Centro de Investigaciones Energéticas, Medioambientales y Tecnológicas, CIEMAT, 28040, Madrid, Spain

${ }^{i}$ Department of Physics, Drexel University, Philadelphia, Pennsylvania 19104, U.S.A.

${ }^{j}$ Institute of Nuclear Research of the Russian Academy of Sciences, Moscow 117312, Russia

${ }^{k}$ IRFU, CEA, Université Paris-Saclay, 91191 Gif-sur-Yvette, France

${ }^{l}$ Department of Physics, Kitasato University, Sagamihara, 252-0373, Japan

${ }^{m}$ Department of Physics, Kobe University, Kobe, 657-8501, Japan

${ }^{n}$ NRC Kurchatov Institute, 123182 Moscow, Russia

${ }^{\circ}$ Max-Planck-Institut für Kernphysik, 69117 Heidelberg, Germany

${ }^{p}$ University of Notre Dame, Notre Dame, Indiana 46556, U.S.A.

${ }^{q} I P H C$, CNRS/IN2P3, Université de Strasbourg, 67037 Strasbourg, France

${ }^{r}$ SUBATECH, CNRS/IN2P3, Université de Nantes, IMT-Atlantique, 44307 Nantes, France

${ }^{s}$ Research Center for Neutrino Science, Tohoku University, Sendai 980-8578, Japan

${ }^{t}$ Department of Physics, Tokyo Institute of Technology, Tokyo, 152-8551, Japan

${ }^{u}$ Department of Physics, Tokyo Metropolitan University, Tokyo, 192-0397, Japan

${ }^{v}$ Physik Department, Technische Universität München, 85748 Garching, Germany

${ }^{w}$ Kepler Center for Astro and Particle Physics, Universität Tübingen, 72076 Tübingen, Germany

${ }^{y}$ Universidade Estadual de Campinas-UNICAMP, Campinas, SP, 13083-970, Brazil

${ }^{z}$ Center for Neutrino Physics, Virginia Tech, Blacksburg, Virginia 24061, U.S.A.

${ }^{a a}$ LNCA Underground Laboratory, CNRS/IN2P3-CEA, Chooz, France

E-mail: navas@lal.in2p3.fr, pau.novella@ific.uv.es

ABSTRACT: A $\theta_{13}$ oscillation analysis based on the observed antineutrino rates at the Double Chooz far and near detectors for different reactor power conditions is presented. This approach provides a so far unique simultaneous determination of $\theta_{13}$ and the total background rates without relying on any assumptions on the specific background contributions. The analysis comprises 865 days of data collected in both detectors with at least one reactor in operation. The oscillation results are enhanced by the use of 24.06 days (12.74 days) of reactor-off data in the far (near) detector. The analysis considers the $\bar{\nu}_{e}$ interactions up to a visible energy of $8.5 \mathrm{MeV}$, using the events at higher energies to build a cosmogenic background model considering fast-neutrons interactions and ${ }^{9} \mathrm{Li}$ decays. The backgroundmodel-independent determination of the mixing angle yields $\sin ^{2}\left(2 \theta_{13}\right)=0.094 \pm 0.017$, being the best-fit total background rates fully consistent with the cosmogenic background model. A second oscillation analysis is also performed constraining the total background rates to the cosmogenic background estimates. While the central value is not significantly 
modified due to the consistency between the reactor-off data and the background estimates, the addition of the background model reduces the uncertainty on $\theta_{13}$ to 0.015 . Along with the oscillation results, the normalization of the anti-neutrino rate is measured with a precision of $0.86 \%$, reducing the $1.43 \%$ uncertainty associated to the expectation.

KeYwords: Neutrino Detectors and Telescopes (experiments), Oscillation

ArXiv EPrint: 2007.13431 


\section{Contents}

1 Introduction 1

$2 \quad \bar{\nu}_{e}$ selection and expected backgrounds $\quad 2$

3 Reactor-on and reactor-off data samples 5

$\begin{array}{lll}4 & \text { Reactor rate modulation analysis } & 7\end{array}$

$\begin{array}{llr}5 & \theta_{13} \text { and background measurements } & 9\end{array}$

6 Summary and conclusions 13

\section{Introduction}

In the last few decades, neutrinos have been proven to be massive particles in several oscillation experiments [1]. The oscillations among the three active neutrino species are now well established, connecting the mass eigenstates $\left(\nu_{1}, \nu_{2}, \nu_{3}\right)$ with the flavor eigenstates $\left(\nu_{e}, \nu_{\mu}, \nu_{\tau}\right)$. The 3 -flavor neutrino oscillations are described by means of three mixing angles $\left(\theta_{12}, \theta_{23}, \theta_{13}\right)$, two independent mass square differences $\left(\Delta m_{21}^{2}, \Delta m_{31}^{2}\right)$, and one phase responsible for the $C P$-violation in the leptonic sector $\left(\delta_{C P}\right)$. After the observation of the dominant oscillations in the so-called solar $[2,3]$ and atmospheric sectors $[4,5]$, respectively driven by $\left(\theta_{12}, \Delta m_{21}^{2}\right)$ and $\left(\theta_{23}, \Delta m_{31}^{2}\right)$, reactor neutrino experiments have recently observed the oscillation induced by the last mixing angle, $\theta_{13}$. Double Chooz, Daya Bay and RENO have provided precise measurements of $\theta_{13}[6-9]$, relying on the observation of the disappearance of electron antineutrinos $\left(\bar{\nu}_{e}\right)$ generated in nuclear reactors at typical flight distances of $1-2 \mathrm{~km}$. The $\theta_{13}$ value offered by reactor experiments is used as an external constraint in current and future accelerator-based experiments aiming at the measurement of $\delta_{C P}$ (see for instance [10]). As a consequence, reactor neutrino experiments play a major role in the search for the leptonic CP-violation.

Double Chooz and other reactor experiments detect the electron antineutrinos via the $\bar{\nu}_{e} p \rightarrow e^{+} n$ interaction, usually referred to as inverse beta decay (IBD). The time and spatial coincidence of the prompt positron and the delayed neutron capture signals yields a large signal-to-background ratio. However, accidental and correlated events induced by fast neutrons and cosmogenic radio-nuclides can mimic the characteristic IBD signature, becoming non-negligible backgrounds. The oscillation analyses presented in [6-9] are based on background models built assuming a number of background sources. The rate and energy spectrum of each background contribution is estimated from the data collected during reactor-on periods, and incorporated to the total background expectation. Accounting for these background models, $\theta_{13}$ is derived from the observed energy-dependent deficit of $\bar{\nu}_{e}$ with respect to a MC-based null-oscillation expectation or the unoscillated flux measured 
at a near detector. As a consequence, the oscillation analyses are background-modeldependent and the uncertainty on the background expectations may have a non-negligible impact on the uncertainty of $\theta_{13}$.

In this paper, an alternative background-model-independent oscillation analysis is presented: the Reactor Rate Modulation (RRM). In this approach, the comparison of the observed rate of $\bar{\nu}_{e}$ candidates with respect to the expected $\bar{\nu}_{e}$ rate in absence of oscillations is performed for different reactor operation conditions ranging from zero to full thermal power. This allows for a simultaneous determination of both $\theta_{13}$ and the total background rate, without making any consideration about the individual background sources. This technique is particularly competitive in the Double Chooz experiment, as it collects data from only two nuclear cores. In addition, a background-model-dependent result on $\theta_{13}$ is also obtained following the same RRM procedure. In this case, the precision on $\theta_{13}$ is improved by incorporating to the analysis a background model based on [9], providing also a consistency test for the model itself. This paper extrapolates the RRM analysis described in [11], which uses only the far detector of the Double Chooz experiment, to a multi-detector setup considering both the near and far detectors. The oscillation analysis also incorporates for the first time the reactor-off data collected by both detectors during 2017, which offer a constraint to the BG rate and serves as independent validation of the BG model. Beyond the neutrino oscillation, the analysis also yields a measurement of the observed rate of IBD interactions. The value is found to be fully consistent with the antineutrino flux normalization provided by Bugey-4 [12], reducing its associated uncertainty.

This paper is organized as follows. Section 2 describes the selection of the $\bar{\nu}_{e}$ candidates and the corresponding expected backgrounds, while section 3 describes the reactor-on and reactor-off data samples used for the oscillation analysis. Section 4 follows with the definition of the RRM approach and the systematic uncertainties involved. Finally, the oscillation analysis results are presented in section 5 with and without the background model constraint, and section 6 concludes with an overview.

\section{$2 \quad \bar{\nu}_{e}$ selection and expected backgrounds}

The setup of the Double Chooz experiment consists of two identical detectors measuring the $\bar{\nu}_{e}$ flux generated at the two reactors (B1 and B2, with a thermal power of $4.25 \mathrm{GW}$ each) of the Chooz B nuclear power plant, operated by Électricité de France. The average distances between the far (FD) and near (ND) detectors and the reactor cores are $L \sim 400$ $\mathrm{m}$ and $L \sim 1050 \mathrm{~m}$, respectively. Both detectors are identical and yield effectively identical responses after calibration, thus leading to a major reduction of the correlated systematic uncertainties in the oscillation analyses. The detectors consist of a set of concentric cylinders and an outer muon veto on the top. The innermost volume (neutrino target or NT) contains $10 \mathrm{~m}^{3}$ of Gd-loaded (0.1\%) liquid scintillator inside a transparent acrylic vessel. This volume is surrounded by another acrylic vessel filled with $23 \mathrm{~m}^{3}$ of Gd-unloaded scintillator (gamma-catcher or GC). This second volume was originally meant to fully contain the energy deposition of gamma rays from the neutron capture on Gd and the positron annihilation in the target region. The GC is in turn contained within a third volume 
(buffer) made of stainless steel and filled with non-scintillating mineral oil. The surface of the buffer is covered with an array of 390 low background 10-inch PMTs. The NT, GC and buffer tank define the inner detector (ID). The ID is surrounded by the inner muon veto (IV), a $50 \mathrm{~cm}$ thick liquid scintillator volume equipped with 78 8-inch PMTs. Finally, the upper part of the detectors is covered by an outer muon veto (OV), made of plastic scintillator strips grouped in different modules. While the ID is meant to detect the IBD interactions and to allow for the event vertex and energy reconstruction, the IV and OV are devoted to the suppression and rejection of backgrounds.

The IBD candidates selection in the RRM analysis follows the lines described in [9]. The selection relies on the twofold-coincidence signature of the IBD process, providing a prompt trigger $\left(e^{+}\right)$and a delayed trigger (neutron capture). The energy of the prompt signal (visible energy) is directly related to the energy of the interacting $\bar{\nu}_{e}$ : $\mathrm{E}_{\bar{\nu}_{e}} \approx \mathrm{E}_{e^{+}}+0.78 \mathrm{MeV}$. While the Double Chooz detectors were originally designed to exploit the large neutron capture cross-section in Gd and the characteristic de-excitation gammas $(\sim 8 \mathrm{MeV})$, the so-called Total Neutron Capture ( $\mathrm{TnC}$ ) selection approach accounts for neutron captures in $\mathrm{H}$ (see $[13,14]$ ), $\mathrm{C}$ and $\mathrm{Gd}$. This implies that IBD detection volume considers both the NT and the GC, boosting the statistical sample of the $\bar{\nu}_{e}$ candidates by almost a factor of 3 . After a series of muon-induced background vetoes based on the ID, IV and OV data (see [9] for details), a time and spatial correlation between the prompt and delayed signals is required. The time difference between the signals is comprised between $0.5 \mu \mathrm{s}$ and $800 \mu \mathrm{s}$, while the distance between the vertexes is imposed to be below $120 \mathrm{~cm}$. In addition, an artificial neural network (ANN) has been developed relying on the promptdelayed correlation to set a cut reducing the rate of random coincidences, especially in the n-H events (see [14] for details). The energy windows considered for the prompt and delayed signals are $1.0-8.5 \mathrm{MeV}$ and $1.3-10.0 \mathrm{MeV}$, respectively. Unlike in the IBD selection presented in [9], where the prompt energy signal is extended to $20 \mathrm{MeV}$ to better constraint the background shapes, in this analysis it is restricted to be below $8.5 \mathrm{MeV}$ ( $>99.96 \%$ of the reactor $\bar{\nu}_{e}$ ). As discussed below, the IBD candidates above this energy are used to infer a background expectation.

The physical events mimicking the IBD signature have been discussed in [9]. In Double Chooz, given the small overburden of the detectors (depths of $\sim 100 \mathrm{~m}$ and $\sim 30 \mathrm{~m}$ for the FD and ND, respectively), the muon-induced cosmogenic backgrounds dominate. These correspond mostly to fast-neutrons and unstable isotopes produced upon ${ }^{12} \mathrm{C}$ spallation (mainly ${ }^{9} \mathrm{Li}$, as no indication of ${ }^{8} \mathrm{He}$ is reported in [15]). While the fast-neutron twofold signature is due to a proton recoil on $\mathrm{H}$ followed by the $\mathrm{n}$-capture, ${ }^{9} \mathrm{Li}$ undergoes a $\beta$ $\mathrm{n}$ decay. Other cosmogenic backgrounds (like $\mu$ decay at rest or ${ }^{12} \mathrm{~B}$ ) are estimated to be negligible. Apart from the correlated backgrounds, random coincidences of natural radioactivity events and neutron captures (hereafter, accidental background) also become a non-negligible contamination in the IBD candidates samples. However, the estimation of the accidental background contribution relying on the rate of single events is very precise $(<0.5 \%$ in the FD and $0.1 \%$ in the ND). The reactor-on-based background model adopted in the current analysis is built with the contributions of fast-neutrons, ${ }^{9} \mathrm{Li}$ and accidental events. The only difference with respect to the model in [9] is the range of the visible energy 

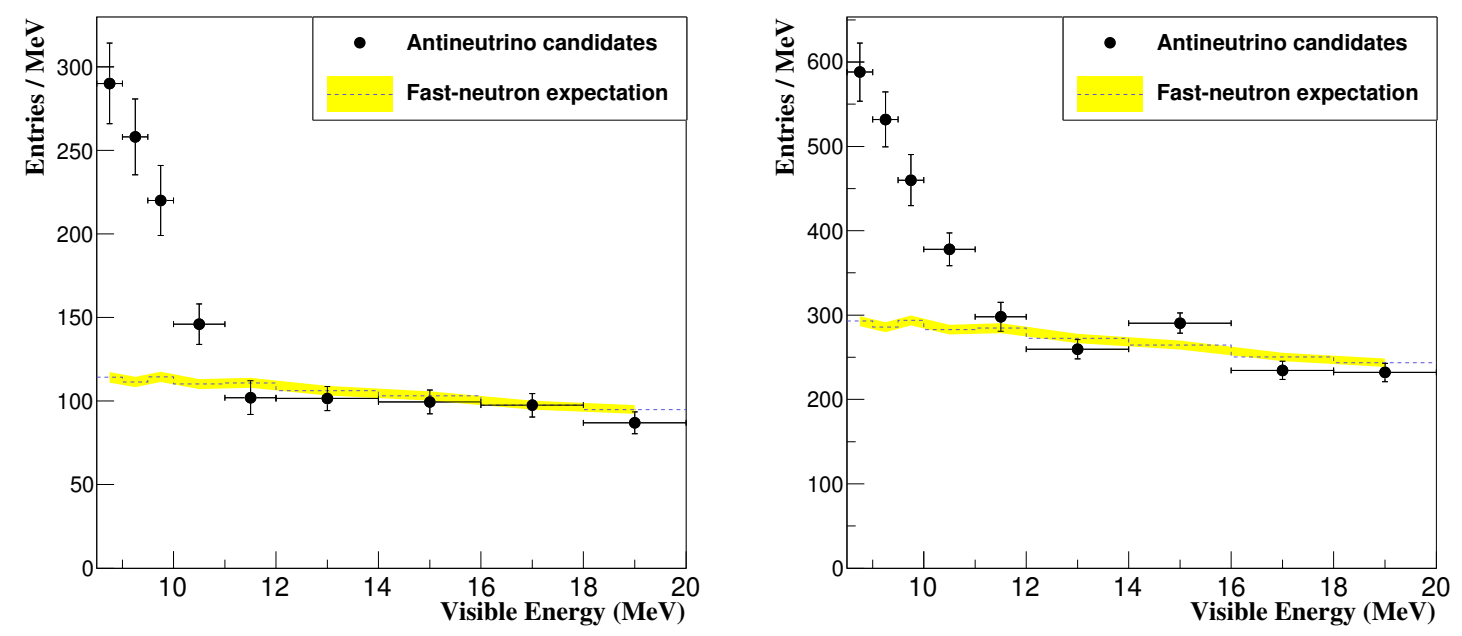

Figure 1. IBD candidates (black dots) as a function of the visible energy above $8.5 \mathrm{MeV}$, extracted from reactor-on data in the FD (left) and the ND (right). The expected contribution from fastneutrons is shown with dashed line and yellow error band. The fast-neutron subtraction in the 8.5-12 MeV range provides the number of ${ }^{9} \mathrm{Li}$ decays which is used to extrapolate the total rate below $8.5 \mathrm{MeV}$.

\begin{tabular}{|cccc|}
\hline Rate $\left(\right.$ day $\left.^{-1}\right)$ & FD (SD) & FD (MD) & ND (MD) \\
\hline Accidental & $3.930 \pm 0.010$ & $4.320 \pm 0.020$ & $3.110 \pm 0.004$ \\
Fast-neutron & $1.09 \pm 0.03$ & $8.89 \pm 0.18$ \\
${ }^{9}$ Li isotope & $2.30 \pm 0.30$ & $14.09 \pm 1.62$ \\
\hline
\end{tabular}

Table 1. Background expectation in the $1.0-8.5 \mathrm{MeV}$ window. The accidental, fast-neutron and ${ }^{9} \mathrm{Li}$ decay contributions to the background model are derived from reactor-on IBD candidates.

and the estimation of the ${ }^{9} \mathrm{Li}$ contribution from the candidates observed in the $8.5 \mathrm{MeV}$ to $12.0 \mathrm{MeV}$ energy window. The fast-neutron rate and energy spectrum is measured from events tagged by the IV up to $20 \mathrm{MeV}$. Subtracting the fast-neutron contribution in the 8.5-12.0 MeV range, as shown in figure 1 , offers a direct measurement of the number of ${ }^{9} \mathrm{Li}$ decays (the $\bar{\nu}_{e}$ contribution is $0.030 \pm 0.009 \%$ ). Given that the spectral shape of the ${ }^{9} \mathrm{Li}$ prompt signal is well known [15], the fraction of the spectrum below (above) $8.5 \mathrm{MeV}$ is computed to be $89.3 \pm 0.5 \%(10.7 \pm 0.5 \%)$. This number allows to extrapolate the total number of ${ }^{9} \mathrm{Li}$ decays in the $1.0-8.5 \mathrm{MeV}$ energy window considered for the RRM oscillation analysis. The expected rates for the fast-neutron, ${ }^{9} \mathrm{Li}$ and accidental backgrounds are summarized in table 1 . The background estimates are quoted separately for the first phase of the experiment (single-detector, hereafter SD), operating only the FD, and the second phase (multi-detector, hereafter MD) with both detectors running. The increase in the FD accidental rate between both periods is due to the increase of the light noise background described in [16]. This noise has been suppressed in the ND covering the PMT bases with a radioupure polyester film, yielding a reduction in the accidental rate with respect to the FD. The increase in the FD accidental rate uncertainty is due to the smaller statistical sample of random coincidences used to estimate this background in the MD phase. 


\section{Reactor-on and reactor-off data samples}

The Double Chooz data have been taken under different reactor operating conditions. In particular, the total $\bar{\nu}_{e}$ flux changes significantly during the reactor refuelling periods, when only one of the cores is in operation. In addition, the flux depends on the cores fuel composition, thus evolving in time. As done in [9], the oscillation analysis presented in this work comprises the data taken between April 2011 and January 2013 (481 days), when only the FD was available, and between January 2015 and April 2016 (384 days), when the FD and the ND were simultaneously collecting data. According to the selection described in section 2 , the number of $\bar{\nu}_{e}$ candidates (actual $\bar{\nu}_{e}$ plus background contributions) in the single-detector period is 47351 , while in the multi-detector period is respectively $42054 \mathrm{in}$ the FD and 206981 in the ND. The time evolution of the candidates rate for the MD data samples is shown in figure 2, where the days with one (1-Off) and two (2-On) reactors in operation are clearly visible. A prediction of the unoscillated $\bar{\nu}_{e}$ flux during the reactoron periods has been carried out as described in previous Double Chooz publications [6]. The reactor flux model is adopted from $[17,18]$. While the ${ }^{235} \mathrm{U},{ }^{239} \mathrm{Pu}$, and ${ }^{241} \mathrm{Pu}$ isotopes contributions are derived from the Institut Laue-Langevin (ILL) reactor data (see for instance [19]), the contribution from ${ }^{238} \mathrm{U}$ is predicted from [20]. The time evolution of the fission fractions are accounted for using dedicated Chooz reactor simulations. As done in past Double Chooz publications where the ND was not available, Bugey4 [12] data have been used as a virtual near detector to define the absolute flux normalization in SD. Although this is not required in oscillation analyses with MD data, the associated flux simulation still accounts for the Bugey4-based normalization in order to keep the consistency between the SD and MD flux expectations. This choice does not impact the oscillation analysis precision as the Bugey-4 uncertainty (1.4\%) is fully correlated between the FD and the ND, and therefore suppressed to a negligible level in a multi-detector analysis.

Among the $\theta_{13}$ reactor-based oscillation experiments, Double Chooz is unique in obtaining reactor-off data (2-Off) when the two cores of the Chooz site are brought down for refuelling or maintenance. Since the Daya Bay and RENO experiments are exposed to the $\bar{\nu}_{e}$ fluxes from 6 different cores, they have been so far unable to collect data when all of them are off. Double Chooz has taken reactor-off data samples during both the SD (two samples in 2011 and 2012) and MD (two samples in 2017) periods. The corresponding livetimes and number of IBD candidates in each period and detector are listed in table 2 . In order to reduce the cosmogenic backgrounds, a time veto of $1.25 \mu \mathrm{s}$ is applied after each detected muon. Thus, the corresponding livetimes differ in the near and far detectors during the MD period due to the different overburdens and muon rates in the experimental sites. Applying the selection cuts to the reactor-off data provides an inclusive sample of the different backgrounds, regardless of their origin, with a contribution from residual antineutrinos. After the nuclear reactors are turned off, $\beta$ decays from fission products keep taking place generating a residual flux of $\bar{\nu}_{e}$ which vanishes with time. Since the reactoroff periods are short in time, the contribution from the residual $\bar{\nu}_{e}$ flux is small but not negligible. However, the amount of residual $\bar{\nu}_{e}$ can be estimated either by means of Monte Carlo simulations, or by performing a relative comparison of the rates observed at different 


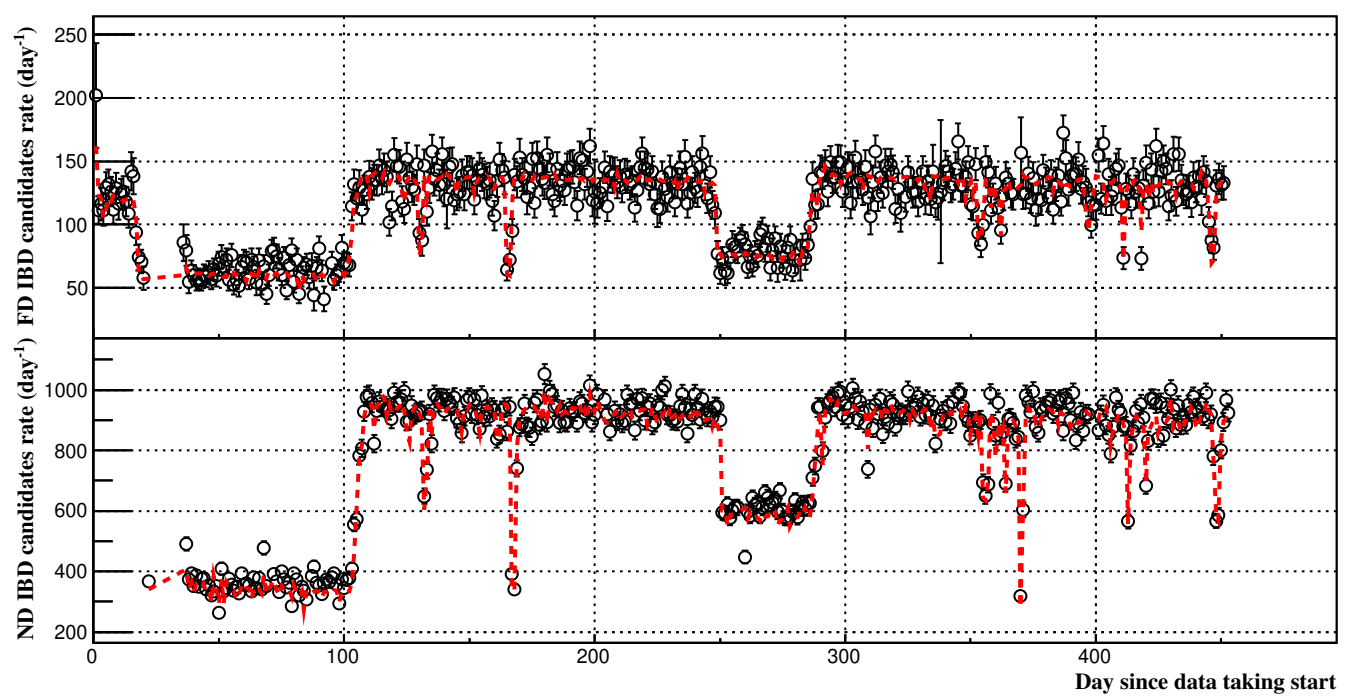

Figure 2. IBD candidates rate for FD (top) and ND (bottom) as a function of the day of data taking during the MD period. Empty circles show the observed rate of candidates, while the dashed red line shows the expected unoscillated $\bar{\nu}_{e}$ rate according to the reactor-on simulation. The significant rate difference in the various 1-Off periods for each detector corresponds to the different baselines between the detector and the two reactors.

\begin{tabular}{|cccc|}
\hline Detector (period) & FD (SD) & FD (MD) & ND (MD) \\
\hline Live time (day) & 7.16 & 16.90 & 12.74 \\
IBD Candidates $\left(\right.$ day $\left.^{-1}\right)$ & $7.96 \pm 1.05$ & $7.99 \pm 0.69$ & $29.91 \pm 1.53$ \\
\hline Expected background $\left(\right.$ day $\left.^{-1}\right)$ & $7.32 \pm 0.30$ & $7.71 \pm 0.30$ & $26.09 \pm 1.63$ \\
\hline
\end{tabular}

Table 2. Reactor-off data samples during SD and MD. The last row shows the total background expectation between 1.0 and $8.5 \mathrm{MeV}$ according to the model described in section 2, without considering the residual $\bar{\nu}_{e}$.

baselines. Thereby once the residual neutrinos are estimated, the reactor-off data allow for a direct measurement of the total background remaining in the $\bar{\nu}_{e}$ candidates samples.

The SD reactor-off data has been used in [21] to estimate the total background rate in previous Double Chooz IBD selection procedures, as well as to confront it with the corresponding background models. In this analysis, the data have been reprocessed with the current IBD selection, yielding the rate of candidates quoted in table 2. In order to estimate the residual neutrino contribution, the Monte-Carlo approach described in [21] has been adopted. A dedicated simulation has been performed with FISPACT [22], an evolution code predicting the isotope inventory in the reactor cores. The neutrino spectrum is then computed using the BESTIOLE [18] database. The expected rate of residual $\bar{\nu}_{e}$ in SD reactor-off period is found to be $0.58 \pm 0.18$ day $^{-1}$. Once subtracted to the observed rate of events, the measured inclusive background rate is $7.38 \pm 1.07$ day $^{-1}$, in good agreement with the expectation from the background model defined in section 2. 

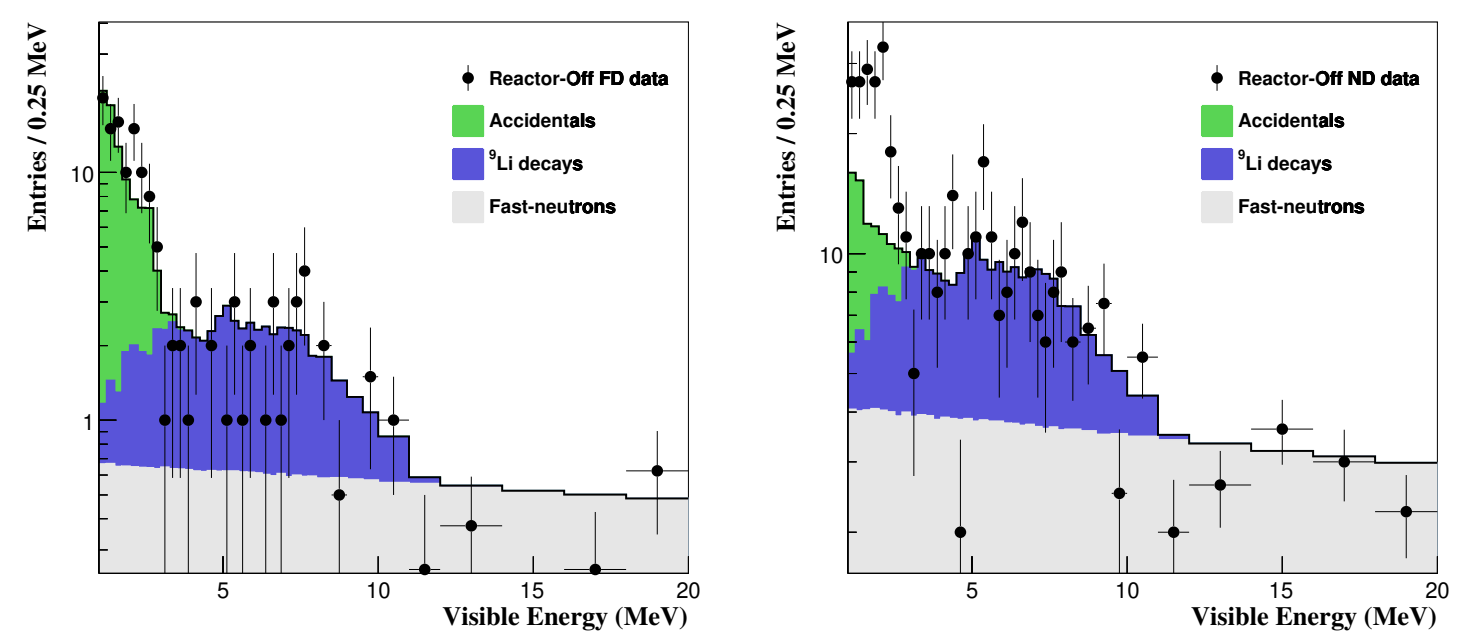

Figure 3. Reactor-off IBD candidates as a function of the visible energy during the MD period. The observed candidates in the FD (left) and the ND (right) are superimposed to the background model described in [9], considering accidental coincidences, fast-neutrons and ${ }^{9} \mathrm{Li}$ decays.

The larger reactor-off statistical sample in the MD period, especially in the ND, allows for detailed comparisons with the background model adopted in this work and described in [9]. The energy spectra of the reactor-off IBD candidates in the FD and ND are shown in figure 3, superimposed to the background model. The expectation reproduces the data at high energies, but deviates below $\sim 3.0 \mathrm{MeV}$. This discrepancy corresponds to the presence of the residual neutrinos, whose spectrum is known to vanish above $3 \mathrm{MeV}$ due to the involved parent isotopes. According to the different geometrical acceptance between the detectors $\left(L_{\mathrm{FD}}^{2} / L_{\mathrm{ND}}^{2} \sim 7\right)$, the contribution of the residual $\bar{\nu}_{e}$ in the ND is significantly larger than in the FD. The observed reactor-off candidates above $8.5 \mathrm{MeV}$ have been used to perform ${ }^{9} \mathrm{Li}$ estimations following the analysis described in section 2 , being the only difference that no IBD interactions are expected. The obtained ${ }^{9} \mathrm{Li}$ estimates up to $8.5 \mathrm{MeV}$ are $(0.52 \pm 1.49) \mathrm{day}^{-1}$ in the FD and $(9.33 \pm 5.28) \mathrm{day}^{-1}$ in the ND, consistently with the reactor-on expectations quoted in table 1.

\section{Reactor rate modulation analysis}

The measurement of the mixing angle $\theta_{13}$ in reactor experiments relies on the comparison of the observed prompt energy spectrum with the expectation in absence of oscillation. Both the deficit in the observed number of candidates and the distortion of the energy spectrum are accounted for in a rate plus shape $(\mathrm{R}+\mathrm{S})$ analysis, as done in [9]. The null-oscillation expectation comes either from a reactor flux simulation or from the measurement of the flux at a short baseline (near detector), where the oscillation effect is small or still negligible. Besides the $\bar{\nu}_{e}$ flux, a $\mathrm{R}+\mathrm{S}$ background model in each detector is also considered, assuming a certain number of background sources. Thus, the $\theta_{13}$ determination becomes backgroundmodel-dependent and the associated uncertainty contains a non-negligible contribution from the background model. 
As an alternative approach, the RRM analysis offers a background-model-independent measurement of $\theta_{13}$. A rate-only (RO) determination of the mixing angle is implemented by comparing the observed rate of candidates $\left(R^{\text {obs }}\right)$ with the expected one $\left(R^{\exp }\right)$ for different reactor thermal power $\left(P_{\mathrm{th}}\right)$ conditions. As the signal-to-background ratio varies depending on $P_{\text {th }}$ (the $\bar{\nu}_{e}$ flux increases with the thermal power while the background rate remains constant), the RRM analysis provides a simultaneous estimation of $\theta_{13}$ and the total (inclusive) background rate, independently of the number of background sources. Relying on rate-only information, these estimations are not affected by the energy distortion (with respect to the predicted spectrum) first reported in [6] and further discussed in [23]. The simple experimental setup of Double Chooz, collecting 2-On, 1-Off and 2-Off data, boosts the precision of the RRM results by offering a powerful lever arm to constrain the total background. The current analysis implements for the first time a multi-detector RRM technique, using reactor-on and reactor-off data collected with both the FD and the ND. As in $\mathrm{R}+\mathrm{S}$ analyses, the systematic uncertainties are highly suppressed by means of the relative comparison of the observed flux at different detectors. Beyond the error suppression, the comparison of the measurements at both detectors also provides a determination of the residual neutrino rate $\left(R^{\mathrm{r} \nu}\right)$ in the MD reactor-off period. This ensures a precise determination of the total background rate which does not rely on Monte-Carlo simulations for the $R^{\mathrm{r} \nu}$ estimation.

The RRM technique exploits the correlation of the expected and observed rates, which follows a linear model parametrized by $\sin ^{2}\left(2 \theta_{13}\right)$ and the total background rate, $B_{d}$ :

$$
R_{d}^{\mathrm{obs}}=B_{d}+R_{d}^{\mathrm{exp}}=B_{d}+\left(1-\sin ^{2}\left(2 \theta_{13}\right) \eta_{d}^{\mathrm{osc}}\right) R_{d}^{\nu},
$$

where the subindex $d$ stands for either the FD or the ND, $R^{\nu}$ is the expected rate of antineutrinos in absence of oscillation, and $\eta^{\text {osc }}$ is the average disappearance coefficient, $\left\langle\sin ^{2}\left(\Delta m^{2} L / 4 E_{\bar{\nu}_{\mathrm{e}}}\right)\right\rangle$, which differs between reactor-on and reactor-off $\left(R^{\nu}=R^{\mathrm{r} \nu}\right)$ data due to the different $\bar{\nu}_{e}$ energy spectrum. This relation between $R_{d}^{\mathrm{obs}}$ and $R_{d}^{\exp }$ is evaluated for data taken at various reactor conditions, grouping the IBD candidates and the expected $\bar{\nu}_{e}$ in bins of the total baseline-adjusted thermal power $\left(P_{\mathrm{th}}^{*}=\sum_{i}^{\mathrm{N}_{\mathrm{r}}} P_{\mathrm{th}}^{i} / L_{i}^{2}\right.$, where $N_{r}=2$ is the number of cores). A fit of these data points to the model expressed in eq. (4.1) yields the determination of $\sin ^{2}\left(2 \theta_{13}\right)$ and $B_{d}$. As the accidental background rate is known with a precision below $1 \%$, the fit is performed with accidental-subtracted samples. As a consequence, hereafter $B_{d}$ refers to all background sources but the accidental one (in short, cosmogenic background). The $\eta^{\text {osc }}$ coefficient is computed, for each $P_{\mathrm{th}}^{*}$ bin, by means of simulations as the integration of the normalized antineutrino energy spectrum multiplied by the oscillation effect driven by $\Delta m^{2}([24])$ and the distance $L$ between the reactor cores and the detectors. The average $\eta_{\mathrm{FD}}^{\mathrm{osc}}\left(\eta_{\mathrm{ND}}^{\mathrm{osc}}\right)$ value in the MD reactor-on period is computed to be 0.55 (0.11). The FD coefficient obtained for the SD reactor-on period is slightly larger (0.56) since the relative contributions of $\bar{\nu}_{e}$ from the B1 and B2 cores differ. According to the residual neutrino spectrum discussed in section 3 , the average $\eta_{\mathrm{FD}}^{\mathrm{osc}}\left(\eta_{\mathrm{ND}}^{\mathrm{osc}}\right)$ in the reactor-off period is $0.86(0.21)$.

The systematic uncertainties related to the IBD detection and the reactor $\bar{\nu}_{e}$ flux have been described in detail in [9]. The same values apply to $R_{d}^{\exp }$, although only the normal- 
ization uncertainties need to be accounted for. These can be divided in three groups: 1) detection efficiency $\left.\left(\sigma_{\epsilon}\right), 2\right)$ reactor-on $\bar{\nu}_{e}$ flux prediction $\left(\sigma_{\nu}\right)$, and 3) reactor-off $\bar{\nu}_{e}$ flux prediction $\left(\sigma_{\mathrm{r} \nu}\right)$. In turn, these errors can be decomposed into their correlated and uncorrelated contributions among the detectors and the reactor cores. The correlated detection efficiency error in the FD and the ND is $0.25 \%$, while the uncorrelated uncertainties are $0.39 \%\left(\sigma_{\epsilon}^{\mathrm{FD}}\right)$ and $0.22 \%\left(\sigma_{\epsilon}^{\mathrm{ND}}\right)$, respectively. Since the far detector has the same performance during the $\mathrm{SD}$ and $\mathrm{MD}$ periods, $\sigma_{\epsilon}^{\mathrm{FD}}$ is fully correlated in the two data samples. Concerning the reactor-on flux uncertainty, only the thermal power $(0.47 \%)$ and fractional fission rates $(0.78 \%)$ are considered to be fully uncorrelated among reactors, in a conservative approach. This implies that the total correlated reactor error is $1.41 \%$ (fully dominated by the Bugey4 normalization), while the uncorrelated is $0.91 \%\left(\sigma_{\nu}\right)$ for both B1 and B2 cores. As discussed in [11], the uncertainty on $P_{\text {th }}$ depends on the thermal power itself. The $P_{\text {th }}$ error in each $P_{\mathrm{th}}^{*}$ bin is computed according to the same procedure. However, as more of $90 \%$ of the reactor-on data are taken at full reactor power (either with 1 or 2 reactors being in operation), the dependence of $\sigma_{\nu}$ with $P_{\text {th }}$ is negligible. In the MD period, $\sigma_{\nu}$ is fully correlated among the two detectors, while $\sigma_{\nu}$ is conservatively treated as fully uncorrelated between the SD and the MD data. The total correlated normalization error in the expected reactor-on IBD flux $\phi$ in the FD and the ND $\left(\sigma_{\phi}\right)$ yields $1.43 \%$, considering the correlated detection and reactor uncertainties. Finally, the uncertainty on the residual $\bar{\nu}_{e}$ in reactor-off periods is treated differently in the SD and MD samples. For the SD sample, $\sigma_{\mathrm{r} \nu}$ is set to $30 \%$ as estimated in [21]. For the MD samples, no error is considered as the IBD rate normalization of $R^{\mathrm{r} \nu}$ is treated as a free parameter in the oscillation fit.

\section{$5 \quad \theta_{13}$ and background measurements}

The fit of the observed rates for each $P_{\mathrm{th}}^{*}$ bin in each detector is based on a standard $\chi^{2}$ minimization. Apart from the free parameters $\sin ^{2}\left(2 \theta_{13}\right), B_{d}$ and $R^{\mathrm{r} \nu}$ (only for MD), a set of nuisance parameters $\bar{\alpha}$ are introduced in order to account for the different flux and detection uncertainties. The $\chi^{2}$ function consists of reactor-on and reactor-off terms, which in turn are divided into individual terms for the FD (SD), FD (MD) and ND detector data samples. In addition, penalty or pull terms are added to constrain each one of the nuisance parameters to the uncertainties quoted in section 4.

Assuming Gaussian-distributed errors, the reactor-on $\chi^{2}$ for each bin in the detector sample $d$ is defined as:

$$
\chi_{\mathrm{on}, d}^{2}=\left(\frac{1}{\sigma_{d}^{\text {stat }}}\right)^{2}\left(R_{d}^{\mathrm{obs}}-R_{d}^{\exp }\left(1+\alpha^{\phi}+\sum_{r=\mathrm{B} 1, \mathrm{~B} 2}\left(w_{d, r} \alpha_{d, r}^{\nu}\right)+\alpha_{d}^{\epsilon}\right)-B_{d}\right)^{2}
$$

where $\sigma_{d}^{\text {stat }}$ is the statistical error and $\alpha^{\phi}, \alpha_{d, r}^{\nu}$, and $\alpha_{d}^{\epsilon}$ are the nuisance parameters accounting for the uncertainties $\sigma_{\phi}, \sigma_{\nu}$ and $\sigma_{\epsilon}^{d}$, respectively. As $\sigma_{\nu}$ is treated as fully uncorrelated between $\mathrm{SD}$ and $\mathrm{MD}$, specific $\alpha_{d}^{\nu}$ parameters are used in both periods: $\alpha_{\mathrm{FD}(\mathrm{SD}), r}^{\nu} \neq$ $\alpha_{\mathrm{FD}(\mathrm{MD}), r}^{\nu}=\alpha_{\mathrm{ND}, r}^{\nu} \equiv \alpha_{r}^{\nu}$. The weights $w_{d, r}$ account for the relative fraction of antineutrinos generated in the reactor $r$ and detected in the detector $d$. These values are computed 
according to the Monte Carlo simulation considering the reactor powers and the baselines. Due to the low statistics in the reactor-off periods, specially in the SD one, the uncertainty in the sample of selected events is considered to be Poisson-distributed. The reactor-off $\chi^{2}$ is then defined as binned Poisson likelihood following a $\chi^{2}$ distribution:

$$
\chi_{\mathrm{off}, d}^{2}=2\left(N_{d}^{\mathrm{obs}} \ln \frac{N_{d}^{\mathrm{obs}}}{C_{d}+N_{d}^{\mathrm{exp}}\left[1+\alpha_{d}^{\epsilon}+\alpha_{d}^{\mathrm{r} \nu}\right]}+C_{d}+N_{d}^{\exp }\left[1+\alpha_{d}^{\epsilon}+\alpha_{d}^{\mathrm{r} \nu}\right]-N_{d}^{\mathrm{obs}}\right)
$$

where $N^{\text {obs }}$ is the number of observed IBD candidates, $C$ is the number of cosmogenic background events ( $C=B \times T$, being $T$ the reactor-off live time), $N^{\exp }$ is the expected number of antineutrinos $\left(N^{\exp }=R^{\exp } \times T\right)$, and $\alpha_{d}^{\mathrm{r} \nu}$ is the parameter accounting for the error on the residual $\bar{\nu}_{e}$ expectation. While in the SD reactor-off data this parameter is constrained by $\sigma_{\mathrm{r} \nu}$, in the MD reactor-off it is left free, but correlated between the FD and the ND according to the ratio of reactor-averaged baselines $L_{d}: \alpha_{\mathrm{ND}}^{\mathrm{r} \nu}=L_{\mathrm{FD}}^{2} / L_{\mathrm{ND}}^{2} \times \alpha_{\mathrm{FD}}^{\mathrm{r} \nu}$. Finally, The last term of the $\chi^{2}$ incorporates Gaussian pulls for the $\bar{\alpha}$ parameters according to their associated uncertainties:

$$
\chi_{\mathrm{pull}}^{2}=\left(\frac{\alpha^{\phi}}{\sigma_{\phi}}\right)^{2}+\left(\frac{\alpha_{\mathrm{FD}}^{\epsilon}}{\sigma_{\epsilon}^{\mathrm{FD}}}\right)^{2}+\left(\frac{\alpha_{\mathrm{ND}}^{\epsilon}}{\sigma_{\epsilon}^{\mathrm{ND}}}\right)^{2}+\sum_{r}^{N_{r}}\left(\frac{\alpha_{r}^{\nu}}{\sigma_{\nu}}\right)^{2}+\sum_{r}^{N_{r}}\left(\frac{\alpha_{\mathrm{FD}(\mathrm{SD}), \mathrm{r}}^{\nu}}{\sigma_{\nu}}\right)^{2}+\left(\frac{\alpha_{\mathrm{FD}(\mathrm{MD})}^{\mathrm{r} \nu}}{\sigma_{\mathrm{r} \nu}}\right)^{2}
$$

The number of reactor-on bins considered for each data sample $\left(N_{\mathrm{b}}\right)$ has been set according to the available statistics. As done in [11], the SD data is divided in $6 P_{\mathrm{th}}^{*}$ bins, while the MD data in 4 bins for both detectors. The overall $\chi^{2}$ function used for the fit can then be expressed as:

$$
\chi^{2}=\sum_{d}\left(\sum_{i}^{N_{\mathrm{b}}} \chi_{\mathrm{on}, d, i}^{2}+\chi_{\mathrm{off}, d}^{2}\right)+\chi_{\mathrm{pull}}^{2}
$$

The results of the $\left(\sin ^{2}\left(2 \theta_{13}\right), B_{\mathrm{FD}}, B_{\mathrm{ND}}\right)$ fit are shown in figure 4 , in terms of the observed versus the expected rate, and of the $68.4 \%, 95.5 \%$ and $99.7 \%$ confidence level (C.L.) regions. The RRM yields best-fit values of $\sin ^{2}\left(2 \theta_{13}\right)=0.094 \pm 0.017, B_{\mathrm{FD}}=$ $3.75 \pm 0.39 \mathrm{day}^{-1}$ and $B_{\mathrm{ND}}=27.1_{-2.1}^{+1.4} \mathrm{day}^{-1}$, with $\chi^{2} / d o f=11.0 / 14$. The backgroundindependent determination of $\theta_{13}$ is consistent with all previous results form Double Chooz, being the precision competitive with the one achieved by the $\mathrm{R}+\mathrm{S}$ analysis in [9]. The values of the total cosmogenic backgrounds in the FD and ND are also consistent with the sum of the ${ }^{9} \mathrm{Li}$ and fast-neutron background expectations quoted in table 1, respectively, with similar associated errors. The best-fit for the residual neutrinos is brought to the physical limit of 0 , with $R_{\mathrm{FD}}^{\mathrm{r} \nu}<0.64 \mathrm{day}^{-1}$ and $R_{\mathrm{ND}}^{\mathrm{r \nu}}<4.2$ day $^{-1}$ at $90 \%$ C.L.

The precision on $\theta_{13}$ can be improved by introducing the constraint of the cosmogenic background estimates into the fit. The background constraint is added to the $\chi^{2}$ function as two extra Gaussian priors:

$$
\chi_{\mathrm{BG}}^{2}=\left(\frac{B_{\mathrm{FD}}^{\mathrm{exp}}-B_{\mathrm{FD}}}{\sigma_{\mathrm{B}}^{\mathrm{ND}}}\right)^{2}+\left(\frac{B_{\mathrm{ND}}^{\mathrm{exp}}-B_{\mathrm{ND}}}{\sigma_{\mathrm{B}}^{\mathrm{ND}}}\right)^{2}
$$



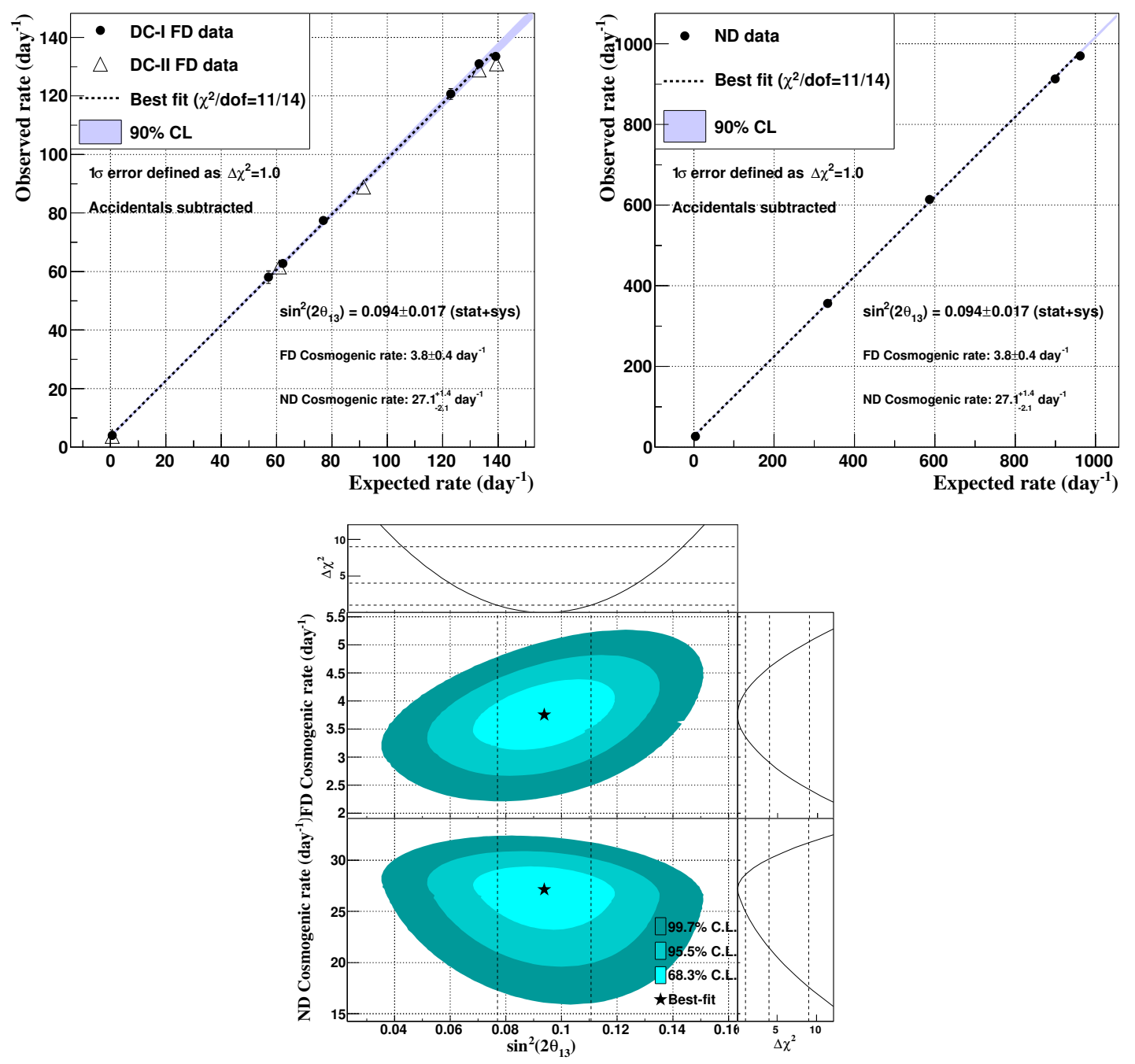

Figure 4. RRM $\left(\sin ^{2}\left(2 \theta_{13}\right), B_{\mathrm{FD}}, B_{\mathrm{ND}}\right)$ fit results. The upper plots show the observed rates versus the expected rates in the FD (left) and the ND (right), superimposed to the best-fit model (dashed line). The statistical errors, not visible, are at the level of $\sim 1 \%(\sim 0.4 \%)$ in the FD (ND). The bottom plot shows the $68.4 \%, 95.5 \%$ and $99.7 \%$ C.L. regions for the three parameters and the one-dimensional projections of $\Delta \chi^{2}$.

where $B^{\exp }$ and $\sigma_{\mathrm{B}}$ stand for the central value and uncertainty of the background expectations. These are built considering the fast-neutron determination from reactor-on data (table 1) and the combination of the ${ }^{9} \mathrm{Li}$ determinations with reactor-on and reactoroff data: $B_{\mathrm{FD}}^{\exp }=3.33 \pm 0.29 \mathrm{day}^{-1}$ and $B_{\mathrm{ND}}^{\exp }=22.57 \pm 1.55 \mathrm{day}^{-1}$. The results of the corresponding $\sin ^{2}\left(2 \theta_{13}\right)$ fit are presented in figure 5 . The fit yields a best-fit value of $\sin ^{2}\left(2 \theta_{13}\right)=0.095 \pm 0.015$, with $\chi^{2} / d o f=13.5 / 16$. As expected due to the consistency between the background estimates and the reactor-off data, the central value is not significantly modified with respect to the background-independent $\theta_{13}$ result. However, the combination of the background model and the reactor-off information allows for a more precise determination of the residual neutrinos, yielding now $R_{\mathrm{FD}}^{\mathrm{r \nu}}=0.48 \pm 0.28 \mathrm{day}^{-1}$ and 

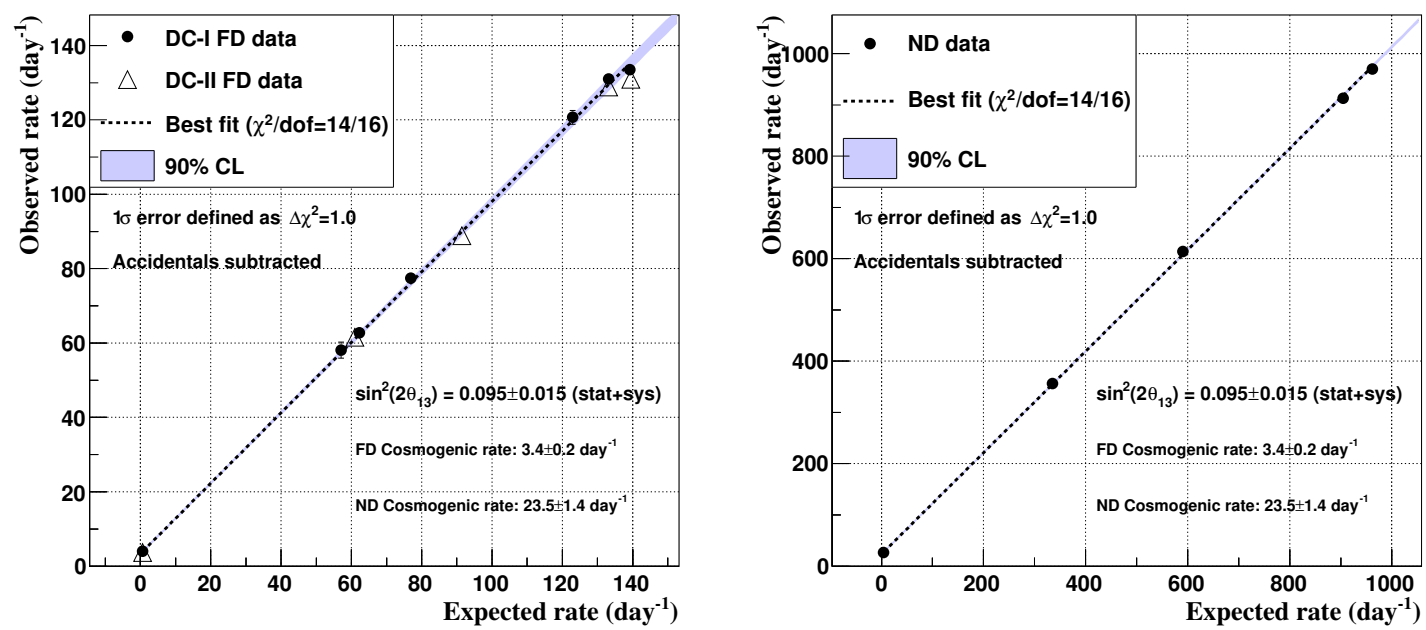

Figure 5. RRM $\sin ^{2}\left(2 \theta_{13}\right)$ fit results. The observed rates versus the expected rates in the FD (left) and the ND (right) are shown superimposed to the best-fit model (dashed line). The statistical errors, not visible, are at the level of $\sim 1 \%(\sim 0.4 \%)$ in the FD (ND). The parameters $B_{F D}$ and $B_{N D}$ are constrained by the estimates obtained from reactor-on data.

\begin{tabular}{|ccc|}
\hline${\text { Background }\left(\text { day }^{-1}\right)}^{-}$ & FD & ND \\
\hline Expectation & $3.33 \pm 0.29$ & $22.57 \pm 1.55$ \\
RRM Best-fit & $3.37 \pm 0.24$ & $23.49 \pm 1.40$ \\
\hline R+S Best-fit & $3.43 \pm 0.25$ & $21.86 \pm 1.33$ \\
\hline
\end{tabular}

Table 3. Cosmogenic background expectations and best-fit values in the FD and the ND. For comparison purposes, the last row shows the best-fit values obtained in the $\mathrm{R}+\mathrm{S}$ analysis [9], restricted to the $1.0-8.5 \mathrm{MeV}$ energy window.

$R_{\mathrm{ND}}^{\mathrm{r \nu}}=3.18 \pm 1.85 \mathrm{day}^{-1}$. According to these non-vanishing values, the best-fit parameters of $B_{\mathrm{FD}}$ and $B_{\mathrm{ND}}\left(3.37 \pm 0.24\right.$ day $^{-1}$ and $23.49 \pm 1.40$ day $^{-1}$, respectively) are slightly reduced with respect to the background-model-independent fit results.

Although fully consistent, the best fit of $B_{\mathrm{ND}}$ is found to be $\sim 7 \%$ higher than the one obtained in [9] $\left(21.86 \pm 1.33 \mathrm{day}^{-1}\right.$ in the $1.0-8.5 \mathrm{MeV}$ energy range). This difference is mostly driven by the background constraint and the use of the new MD reactor-off data, not considered in the $\mathrm{R}+\mathrm{S}$ analysis yet. In table 3 , a summary of the background expectations and the best-fit values is presented. Due to the anti-correlation between $\sin ^{2}\left(2 \theta_{13}\right)$ and the background in the ND (visible in bottom plot of figure 4), the larger best-fit value of $B_{\mathrm{ND}}$ obtained in the current RRM analysis pulls down the central value of $\theta_{13}$ with respect to the $\mathrm{R}+\mathrm{S}$ result $\left(\sin ^{2}\left(2 \theta_{13}\right)=0.105 \pm 0.014\right)$.

Finally, in order to provide a measurement of the IBD rate normalization, the parameter $\alpha^{\phi}$ can be left free in the fit by removing the corresponding pull term in the $\chi^{2}$. Since the correlated detection efficiency is known with a negligible uncertainty $(0.25 \%)$, this parameter provides effectively the relative normalization with respect to the central value of the reactor-on flux simulation. This central value is defined by the mean cross- 
section per fission (see [9] for details), $\left\langle\sigma_{\mathrm{f}}\right\rangle$, measured by Bugey-4. Once corrected for the specific averaged fuel compositions of the Double Chooz reactor cores, it is computed to be $(5.75 \pm 0.08) \times 10^{-43} \mathrm{~cm}^{2} /$ fission. The best-fit value of $\alpha^{\phi}$ yields $0.04 \pm 0.86 \%$, thus being fully consistent with the expectation but reducing the $1.43 \%$ uncertainty on the IBD rate normalization $\sigma_{\phi}$. According to this result, the best-fit of $\sin ^{2}\left(2 \theta_{13}\right)$ is not significantly modified.

\section{Summary and conclusions}

The simple experimental setup of Double Chooz, consisting of only two detectors and two reactors, allows for a simultaneous determination of $\theta_{13}$ and the total background rates. The RRM analysis relies on the rate of observed $\bar{\nu}_{e}$ interactions in data samples collected at different total reactor powers. The comparison of such rates with the null-oscillation Monte Carlo expectations provides a background-independent measurement of $\sin ^{2}\left(2 \theta_{13}\right)$, as well as inclusive background rates in the far and near detectors which do no depend of any a priori assumptions on the individual background sources. This approach is intrinsically different from the usual $\mathrm{R}+\mathrm{S} \theta_{13}$ oscillation analyses implemented in reactor experiments, which are based on background models considering a number of background sources and estimating the corresponding rates and energy spectra from reactor-on data.

In this work, a multi-detector RRM analysis is implemented for the first time. As in $\mathrm{R}+\mathrm{S}$ analyses, the relative comparison of the rates observed at different baselines leads to a major reduction of the involved systematic uncertainties. In particular, the correlated detection and reactor $\bar{\nu}_{e}$ flux errors cancel out, while the uncorrelated flux uncertainty is significantly suppressed. Apart from boosting the precision in the $\theta_{13}$ measurement, the multi-detector measurement of the IBD interactions allows for a determination of the observed IBD rate normalization. The current oscillation analysis also uses for the first time reactor-off data samples for both the FD and ND, offering a powerful handle to constrain the backgrounds. Among the $\theta_{13}$ reactor experiments, Double Chooz is the only one with available reactor-off samples, thus offering a unique cross-check of the background models.

The RRM oscillation fit relies on the minimization of a $\chi^{2}$ function consisting of reactor-on and reactor-off terms, as well as penalty terms constraining the nuisance parameters accounting for the systematic uncertainties to their estimated values. The errors considered in the fit are those impacting the expected IBD rates, namely, the detection efficiency and the reactor flux normalization uncertainties. The $\left(\sin ^{2}\left(2 \theta_{13}\right), B_{\mathrm{FD}}\right.$, $\left.B_{\mathrm{ND}}\right)$ fit yields a background-independent value of $\theta_{13}$ which is consistent with previous Double Chooz results: $\sin ^{2}\left(2 \theta_{13}\right)=0.094 \pm 0.017$. The precision achieved by the $\mathrm{RRM}$ analysis is competitive with that one obtained in the $\mathrm{R}+\mathrm{S}$ fit presented in [9] $\left(\sin ^{2}\left(2 \theta_{13}\right)=0.105 \pm 0.014\right)$, relying on a reactor-on background model. The best fit values of the total cosmogenic background rates in the FD and ND, $B_{\mathrm{FD}}=3.75 \pm 0.39$ day $^{-1}$ and $B_{\mathrm{ND}}=27.1_{-2.1}^{+1.4}$ day $^{-1}$, are also consistent with the background estimates. Thus, these expectations can be added to the RRM in order to improve the precision of the oscillation results: $\sin ^{2}\left(2 \theta_{13}\right)=0.095 \pm 0.015$. The limited reduction on the error is due to the dominant role of the detection and reactor flux systematic uncertainties. The compatibility of the background-model-dependent $\mathrm{R}+\mathrm{S}$ and the $\mathrm{RRM}$ results, as well as the consistency 
between the reactor-off data and the background models, confirms the robustness of the Double Chooz oscillation analyses. Beyond the $\theta_{13}$ result, the RRM fit is used to measure the observed $\bar{\nu}_{e}$ rate normalization. The best-fit value yields a $0.04 \pm 0.86 \%$ deviation with respect to the flux normalization predicted by Bugey-4, thus being fully consistent.

\section{Acknowledgments}

We thank the company Electricité de France (EDF); the European fund FEDER; the Région de Champagne-Ardenne; the Département des Ardennes; and the Communauté de Communes Ardenne Rives de Meuse. We acknowledge the support of the CEA, CNRS/IN2P3, the computer centre CC-IN2P3 and LabEx UnivEarthS in France; the Max Planck Gesellschaft, the Deutsche Forschungsgemeinschaft DFG, the Transregional Collaborative Research Center TR27, the excellence cluster "Origin and Structure of the Universe" and the Maier-Leibnitz-Laboratorium Garching in Germany; the Ministry of Education, Culture, Sports, Science and Technology of Japan (MEXT) and the Japan Society for the Promotion of Science (JSPS) in Japan; the Ministerio de Economía, Industria y Competitividad (SEIDI-MINECO) under grants FPA2016-77347-C2-1-P and MdM-2015-0509 in Spain; the Department of Energy and the National Science Foundation and Department of Energy in the United States; the Russian Academy of Science, the Kurchatov Institute and the Russian Foundation for Basic Research (RFBR) in Russia; the Brazilian Ministry of Science, Technology and Innovation (MCTI), the Financiadora de Estudos e Projetos (FINEP), the Conselho Nacional de Desenvolvimento Científico e Tecnológico (CNPq), the São Paulo Research Foundation (FAPESP) and the Brazilian Network for High Energy Physics (RENAFAE) in Brazil.

Open Access. This article is distributed under the terms of the Creative Commons Attribution License (CC-BY 4.0), which permits any use, distribution and reproduction in any medium, provided the original author(s) and source are credited.

\section{References}

[1] Particle Data Group collaboration, Review of Particle Physics, Phys. Rev. D 98 (2018) 030001 [INSPIRE].

[2] SNO collaboration, Direct evidence for neutrino flavor transformation from neutral current interactions in the Sudbury Neutrino Observatory, Phys. Rev. Lett. 89 (2002) 011301 [nucl-ex/0204008] [INSPIRE].

[3] KAMLAND collaboration, First results from KamLAND: Evidence for reactor anti-neutrino disappearance, Phys. Rev. Lett. 90 (2003) 021802 [hep-ex/0212021] [INSPIRE].

[4] SuPER-KAMIOKANDE collaboration, Evidence for an oscillatory signature in atmospheric neutrino oscillation, Phys. Rev. Lett. 93 (2004) 101801 [hep-ex/0404034] [INSPIRE].

[5] K2K collaboration, Evidence for muon neutrino oscillation in an accelerator-based experiment, Phys. Rev. Lett. 94 (2005) 081802 [hep-ex/0411038] [INSPIRE].

[6] Double CHOOZ collaboration, Improved measurements of the neutrino mixing angle $\theta_{13}$ with the Double CHOOZ detector, JHEP 10 (2014) 086 [Erratum ibid. 02 (2015) 074] [arXiv: 1406.7763] [INSPIRE]. 
[7] DAYA BAy collaboration, Measurement of the Electron Antineutrino Oscillation with 1958 Days of Operation at Daya Bay, Phys. Rev. Lett. 121 (2018) 241805 [arXiv:1809.02261] [INSPIRE].

[8] RENO collaboration, Spectral Measurement of the Electron Antineutrino Oscillation Amplitude and Frequency using 500 Live Days of RENO Data, Phys. Rev. D 98 (2018) 012002 [arXiv: 1610.04326] [INSPIRE].

[9] Double CHOOZ collaboration, Double CHOOZ $\theta_{13}$ measurement via total neutron capture detection, Nature Phys. 16 (2020) 558 [arXiv:1901.09445] [INSPIRE].

[10] T2K collaboration, Constraint on the matter-antimatter symmetry-violating phase in neutrino oscillations, Nature 580 (2020) 339 [Erratum ibid. 583 (2020) E16] [arXiv: 1910.03887] [INSPIRE].

[11] Double CHOOZ collaboration, Background-independent measurement of $\theta_{13}$ in Double CHOOZ, Phys. Lett. B 735 (2014) 51 [arXiv:1401.5981] [InSPIRE].

[12] Y. Declais et al., Study of reactor anti-neutrino interaction with proton at Bugey nuclear power plant, Phys. Lett. B 338 (1994) 383 [INSPIRE].

[13] Double CHOOZ collaboration, First Measurement of $\theta_{13}$ from Delayed Neutron Capture on Hydrogen in the Double CHOOZ Experiment, Phys. Lett. B 723 (2013) 66 [arXiv: 1301.2948] [INSPIRE].

[14] Double CHOOZ collaboration, Measurement of $\theta_{13}$ in Double CHOOZ using neutron captures on hydrogen with novel background rejection techniques, JHEP 01 (2016) 163 [arXiv: 1510.08937] [INSPIRE].

[15] Double CHOOZ collaboration, Yields and production rates of cosmogenic ${ }^{9} \mathrm{Li}$ and ${ }^{8} \mathrm{He}$ measured with the Double CHOOZ near and far detectors, JHEP 11 (2018) 053 [arXiv: 1802.08048] [INSPIRE].

[16] Double CHOOZ collaboration, Characterization of the Spontaneous Light Emission of the PMTs used in the Double CHOOZ Experiment, 2016 JINST 11 P08001 [arXiv:1604.06895] [INSPIRE].

[17] P. Huber, On the determination of anti-neutrino spectra from nuclear reactors, Phys. Rev. C 84 (2011) 024617 [Erratum ibid. 85 (2012) 029901] [arXiv: 1106.0687] [INSPIRE].

[18] T.A. Mueller et al., Improved Predictions of Reactor Antineutrino Spectra, Phys. Rev. C 83 (2011) 054615 [arXiv:1101.2663] [INSPIRE].

[19] K. Schreckenbach, G. Colvin, W. Gelletly and F. Von Feilitzsch, DEtermination of the anti-neutrino spectrum from ${ }^{235} \mathrm{U}$ thermal neutron fission products up to $9.5 \mathrm{MeV}$, Phys. Lett. $B 160$ (1985) 325 [INSPIRE].

[20] N. Haag et al., Experimental Determination of the Antineutrino Spectrum of the Fission Products of ${ }^{238}$ U, Phys. Rev. Lett. 112 (2014) 122501 [arXiv:1312.5601] [INSPIRE].

[21] Double CHOOZ collaboration, Direct Measurement of Backgrounds using Reactor-Off Data in Double CHOOZ, Phys. Rev. D 87 (2013) 011102 [arXiv:1210.3748] [INSPIRE].

[22] R.A. Forrest et al., FISPACT-200\%: user manual, UKAEA-FUS-534 (2007) [http://www.ccfe.ac.uk].

[23] P. Novella, The antineutrino energy structure in reactor experiments, Adv. High Energy Phys. 2015 (2015) 364392 [arXiv:1512.03366] [INSPIRE].

[24] S. Parke, What is $\Delta m_{e e}^{2}$ ?, Phys. Rev. D 93 (2016) 053008 [arXiv:1601.07464] [InSPIRE]. 